\title{
Propuesta de una ecuación lineal para valorar la velocidad de crecimiento somático a partir de la masa corporal de ratas machos Wistar
} Proposal of a linear equation to determine somatic growth velocity by male Wistar rats'

\author{
Marco Antonio Cossio-Bolanos ${ }^{1}$, Rossana Gómez-Campos ${ }^{2,3}$, Silvia Pilco-Quesada ${ }^{4}$, \\ José Luis Lancho-Alonso ${ }^{1}$, Miguel de Arruda ${ }^{5}$ \\ 'Prof. Dr., Laboratorio de Ciencias morfofuncionales, FM, Universidad de Córdoba, España. \\ ${ }^{2}$ Doctoranda de la Facultad de Educación Física, UNICAMP, São Paulo, Brasil. \\ ${ }^{3}$ Facultad de Ciencias de la Actividad Física y Deporte, Universidad Católica de Valencia, España. \\ ${ }^{4}$ Maestrista de la Facultad de Ingeniería de Alimentos, Departamento de Ciencias de Alimentos, UNICAMP, São Paulo, Brasil. \\ ${ }^{5}$ Prof. Dr., Facultad de Educación Física, UNICAMP, São Paulo, Brasil.
}

\begin{abstract}
Resumen
Introducción: Para conocer el grado de maduración biológica de los roedores es necesaria la aplicación de métodos invasivos y no-invasivos, para lo cual los estudios que engloban diversas cepas de ratas necesitan de procedimientos simples de manera de controlar los efectos de confusión que la maduración biológica pudiera ocasionar. Objetivos: Determinar la velocidad de crecimiento (VC) de ratas machos Wistar a partir de la masa corporal y proponer ecuaciones de regresión lineal para predecir el pico de velocidad de crecimiento (PVC). Diseño: Estudio longitudinal de cohorte. Institución: Universidad Estadual de Campinas, São Paulo, Brasil, Departamento de Farmacología. Métodos: De un total de 101 ratas, se seleccionó de forma probabilística-aleatoria (tablas aleatorias) 25 ratas machos Wistar de 3 semanas de vida (21 días de edad). Se evaluó la masa corporal (g) cada semana, hasta las 16 semanas de vida. Para el análisis estadístico se utilizó la estadística descriptiva de la media aritmética (X) y desviación estándar (DE). Para determinar las diferencias significativas entre las edades se usó ANOVA para medidas repetitivas $(p<0,001)$ y para correlacionar las variables de edad, peso y PVC se utilizó $(r)$ de Pearson $(p<0,001)$. La predicción del PVC se efectuó a través del análisis de regresión lineal simple y múltiple StepWise $(p<0,001)$. Principales medidas de resultados: Velocidad de crecimiento; propuesta de ecuaciones de regresión lineal para predecir el pico de velocidad de crecimiento. Resultados: Los resultados muestran que la velocidad de crecimiento evaluada a través de la masa corporal se presentó alrededor de los 42 días de vida. Con dichos resultados se generó tres ecuaciones de regresión lineal simple y múltiple, donde el coeficiente de determinación $\left(R^{2}\right)$ para el modelo 1 fue 0,99 , para el modelo 2, 0,89 y para el modelo 3, 0,99, respectivamente. Estas ecuaciones permiten predecir la proximidad y el alejamiento del PVC de ratas machos Wistar y los resultados pueden ser interpretados a través de 14 niveles. Conclusiones: El PVC se produjo a los 42 dias de vida y las ecuaciones de regresión lineal generadas a partir del peso corporal y la edad permitieron predecir el grado de maduración somática de ratas machos Wistar.
\end{abstract}

Palabras clave: Ratas Wistar, crecimiento, modelos lineales.

Abstract

Introduction: In order to determine the degree of biological maturation in rodents it is necessary to apply non-invasive and invasive methods, for which studies covering different strains of rats need simple procedures to control the confounding effects of biological maturation they may cause. Objectives: To determine from body mass growth velocity of Wistar male rats and to propose linear regression equations to predict growth velocity peak (GVP). Design: Longitudinal cohort study. Institution: State University of Campinas, São Paulo, Brazil, Department of Pharmacology. Methods: From 101 rats 25 male Wistar rats 21 days old were selected in probabilistic-random (randomized tables). Body mass ( $\mathrm{g}$ ) was assessed every week until 16 weeks of life. For statistical analysis, descriptive statistics were used in arithmetic average $(X)$ and standard deviation (SD). To determine significant differences between ages ANOVA for repeated measures was used $(p<0.001)$ and to correlate age variables weight and GVP Pearson $(r)$ was used ( $p$ $<0.01)$. GVP prediction was carried out through simple linear regression analysis and multiple stepwise $(p<0.001)$. Main outcome measures: Growth speed; proposal of linear regression equations to predict growth velocity peak. Results: Growth velocity peak assessed by body mass appeared at about 42 days of life. Three equations and multiple linear regression were generated, where determination of $\left(R^{2}\right)$ coefficient for model 1 was 0.99 , for model 20.89 and model 30.99 , respectively. These equations predicted the proximity and separation of male Wistar rats GVP and results could be interpreted through 14 levels. Conclusions: GVP occurred at 42 days of life and linear regression equations were generated from body weight and age in order to predict somatic maturation degree of male Wistar rats.

Key words: Rats/Wistar, growth, linear models.

An Fac med. 2012;73(2):93-100 


\section{INTRODUCCIÓN}

El crecimiento físico de todo organismo viviente implica un proceso dinámico a lo largo de la vida. Malina y Bouchard ${ }^{(1)}$ consideran que este fenómeno ocurre desde la concepción hasta la muerte, siendo definido como el aumento en el número y tamaño de las células que componen los diversos tejidos del $\operatorname{organismo}^{(2,3)}$, fruto de la hiperplasia (aumento en el número de células), hipertrofia (aumento en el tamaño de células) y la agregación (capacidad de los substratos intercelulares en agregar las células) ${ }^{(1,4)}$. Tales procesos de crecimiento ya fueron alertados tiempo atrás por Enesco y Leblond ${ }^{(2)}$, al investigar el crecimiento físico en ratas; dichos autores consideraron tres fases: la fase inicial denominada hiperplasia, que comprende los primeros 17 días de edad; la fase intermedia (hiperplasiahipertrofia), que va desde los 17 hasta los 48 días de edad; y la fase final (almacenamiento), que comprende desde los 48 hasta los 160 días, aproximadamente.

Dicho proceso de crecimiento puede ser cuantificado, según Prieto y Álvarez ${ }^{(5)}$, mediante la curva de crecimiento, en la que se representa la masa corporal en función del tiempo. Esta forma de cuantificar variables somáticas permite conocer la evolución del crecimiento somático al igual que en otras especies, mostrando por lo general una curva sigmoidea. De hecho, el modelo animal es considerado como una importante herramienta para el estudio de las condiciones que afectan a los seres humanos y que pueden ser simuladas en ratas ${ }^{\left({ }^{6}\right)}$. Esto en razón del uso común de los animales como modelos experimentales de investigación en los laboratorios ${ }^{(7)}$, como, por ejemplo, en fisiología reproductiva ${ }^{(8)}$, en patologías nutricionales ${ }^{(9,10)}$, en investigaciones con restricción alimentaria ${ }^{(11)}$, es decir, en las ciencias de la vida, como la medicina, veterinaria, biología y farmacología ${ }^{(12)}$, respectivamente.

En consecuencia, el sexo, la masa corporal y la edad son factores internos que deben ser considerados y mantenidos constantes durante los experimentos con el fin de conseguir resultados reproducibles ${ }^{(13)}$, sobre todo, cuando se trata de investigaciones que aplican diseños experimentales (pre y post test) en ratas en pleno proceso de crecimiento somático. De esa forma, es necesario conocer las fases del proceso de crecimiento, desarrollo y maduración de las ratas, dado que en el momento de transferir y/o extrapolar los resultados al modelo humano estos deberían ocurrir en las mismas etapas de crecimiento y desarrollo y en las mismas condiciones somáticas. En este sentido, el objetivo del presente estudio fue determinar la velocidad de crecimiento (VC) de ratas machos Wistar a partir de la masa corporal y proponer ecuaciones de regresión lineal para predecir el pico de velocidad de crecimiento (PVC).

\section{MÉTODOS}

El estudio fue del tipo descriptivo de corte longitudinal ${ }^{(14)}$, a partir del cual se estudió un grupo específico (cohorte) durante el tiempo ${ }^{(15)}$, realizando 14 evaluaciones de la masa corporal (cada semana), hasta completar las 16 semanas de vida. Para tal efecto, de un total de 101 ratas se seleccionó de forma probabilística-aleatoria (tablas aleatorias) 25 ratas machos Wistar de 3 semanas (21días de edad), después del período del destete, provenientes del Bioterio del Departamento de Farmacología de la Universidad Estadual de Campinas (UNICAMP) Sao Paulo, Brasil. Los animales fueron alojados en cajas colectivas de plástico (5 ratas por caja) en ciclo claro/oscuro (12h/12h), en ambiente con temperatura constante $\left(23^{\circ} \mathrm{C} \pm 2^{\circ} \mathrm{C}\right)$, alimentados libremente con ración y agua ad líbitum y tratados bajo los principios éticos de experimentación animal según el COBEA (Colegio Brasileiro de Experimentación Animal) y aprobado por el Comité de Ética de la Facultad de Medicina de la Universidad Estadual de Campinas UNICAMP, protocolo $n^{\circ}$. 1914-1. Todo el proceso de recolección de los datos se efectuó durante los meses de enero a mayo de 2009.

Para la evaluación de la masa corporal (g) de las ratas machos, se utilizó una balanza analítica de marca Scaltec modelo SAC-62, con una precisión de (10-4gramos). El procedimiento consistió en colocar los animales sobre la balanza y evaluar la masa corporal en gramos (g) sin que el animal se moviera constantemente. Esta técnica fue repetida por dos veces en el momento de la evaluación, manteniendo siempre los cuidados necesarios de interferencias de objetos extraños y realizando la correspondiente calibración de la balanza a cada 3 evaluaciones, de acuerdo con las sugerencias descritas por CossioBolanos y col ${ }^{(16)}$.

La masa corporal (g) fue evaluada dos veces, con el objetivo de observar el error técnico de medida (ETM) intraevaluador $\sqrt{d}^{2} / 2 \mathrm{n}$. La realización de esta técnica permitió verificar la variabilidad de las medidas (masa corporal) y sirve como un importante indicador de estabilidad. A su vez, con el objetivo de medir la reproductibilidad de la variable de la masa corporal (g) se utilizó el coeficiente de correlación productomomento de Pearson ( $r) \quad(p<0,001)$, como lo sugieren algunos estudios ${ }^{(17,18)}$. Los resultados del ETM y el coeficiente de Pearson pueden ser observados en la tabla 1.

La evaluación de la velocidad del crecimiento somático se refiere a la tasa de crecimiento de un individuo en un lapso determinado; requiere de dos mediciones como mínimo, para lo cual se utiliza la siguiente fórmula básica:

$$
V C=\frac{M C A-M C A N}{E A D-E A N D}=g / d i ́ a
$$

Donde: $\mathrm{MCA}=$ masa corporal actual, MCAN = masa corporal anterior, $E A D=$ edad actual en días y EAND = edad anterior en días.

Los resultados del estudio fueron procesados por medio de la estadística descriptiva de media aritmética $(\mathrm{X})$ y desviación estándar (DE). Para determinar las diferencias significativas de la 
masa corporal entre las edades se utilizó ANOVA para medidas repetidas, con una probabilidad de $(\mathrm{p} \leq 0,001)$. Para relacionar las variables de masa corporal, edad y el pico de velocidad de crecimiento (PVC) se usó el coeficiente de correlación de Pearson (r) $(\mathrm{p}<0,001)$. Con relación a la inferencia estadística, se utilizó el análisis de regresión lineal simple y múltiple StepWise con el objetivo de determinar el nivel predictivo del pico de velocidad de crecimiento (PVC). El análisis de distribución normal de los valores fue verificado por la prueba de Kolmogorov-Smirnov, determinándose distribución normal de los datos.

Para validar las ecuaciones de predicción, se usó la concordancia con el enfoque de componentes de la varianza, por medio del método de Bland y Altman ${ }^{(19)}$; y para verificar las diferencias entre los valores originales del estudio y los valores predichos se aplicó la prueba t para muestras pareadas, con una probabilidad de $(\mathrm{p}<0,001)$. Todo el procesamiento estadístico se analizó por medio del programa Sigma Esta $8,0{ }^{(20)}$.

\section{RESULTADOS}

Los valores de estabilidad y reproductibilidad de las medidas de la masa corporal (g) realizadas a lo largo de las 14 semanas de investigación muestran excelentes valores. Los resultados pueden ser observados en la tabla 1 , donde se distingue que el error técnico de medida ETM es inferior a 0,79\%, así como el coeficiente de correlación de Pearson muestra una alta capacidad de reproductibilidad $(\mathrm{r}=0,99)$.

Durante la monitorización de la masa corporal ( $\mathrm{g}$ ) de las ratas se observó una tendencia al aumento de la masa corporal durante las 14 semanas de estudio, es decir, desde la semana 3 hasta la semana 16. En la figura 1 puede distinguirse el punto de inflexión que se presenta en la semana seis, el cual, da lugar al PVC. Esta tendencia permite obtener una curva lineal du-
Tabla 1. Error técnico de medida intraevaluador (ETM) y el coeficiente de reproductibilidad (r) de la masa corporal (g) de ratas machos Wistar evaluados durante 14 semanas $(n=25)$.

\begin{tabular}{ccccc} 
Edad semanas & Edad días & ETM & $r$ & EEE \\
3 & 21 & 0,707107 & 0,99119 & 0,00350746 \\
4 & 28 & 0,83666 & 0,99486 & 0,00205112 \\
5 & 35 & 0,69282 & 0,99795 & 0,00081834 \\
6 & 42 & 0,707107 & 0,99814 & 0,00074373 \\
7 & 49 & 0,860233 & 0,99881 & 0,00047568 \\
8 & 56 & 0,69282 & 0,99891 & 0,00043594 \\
9 & 63 & 0,787401 & 0,99832 & 0,00067254 \\
10 & 70 & 0,72111 & 0,99893 & 0,00042613 \\
11 & 77 & 0,707107 & 0,99897 & 0,00041279 \\
12 & 84 & 0,69282 & 0,99914 & 0,00034340 \\
13 & 91 & 0,707107 & 0,99941 & 0,00023544 \\
14 & 98 & 0,663325 & 0,99917 & 0,00033146 \\
15 & 105 & 0,692219 & 0,9991 & 0,00036133 \\
16 & 112 & 0,797724 & 0,9990 & 0,00039916 \\
\hline
\end{tabular}

rante el proceso de crecimiento somático de los animales. A su vez, se puede observar que el mayor aumento de la masa corporal (g) fue de la quinta para la sexta semana $(52,5 \mathrm{~g})$. Este aumento es identificado claramente en la figura 2, donde el primer pico se produce de los 35 para los 42 días de vida y el segundo pico en menor proporción a los 63 días, aproximadamente. Por lo tanto, el primer pico representa el $46 \%$

del crecimiento somático en relación al peso adulto.

Por otro lado, en la tabla 2 se observa los valores de ANOVA para medidas repetitivas. Los resultados muestran diferencias significativas $(\mathrm{p}<0,001)$ de masa corporal (g), cuando fueron comparados en función de la edad desde la semana 3 hasta la semana 16 . A su vez, se observa el porcentaje de crecimiento somático (masa corporal) desde los

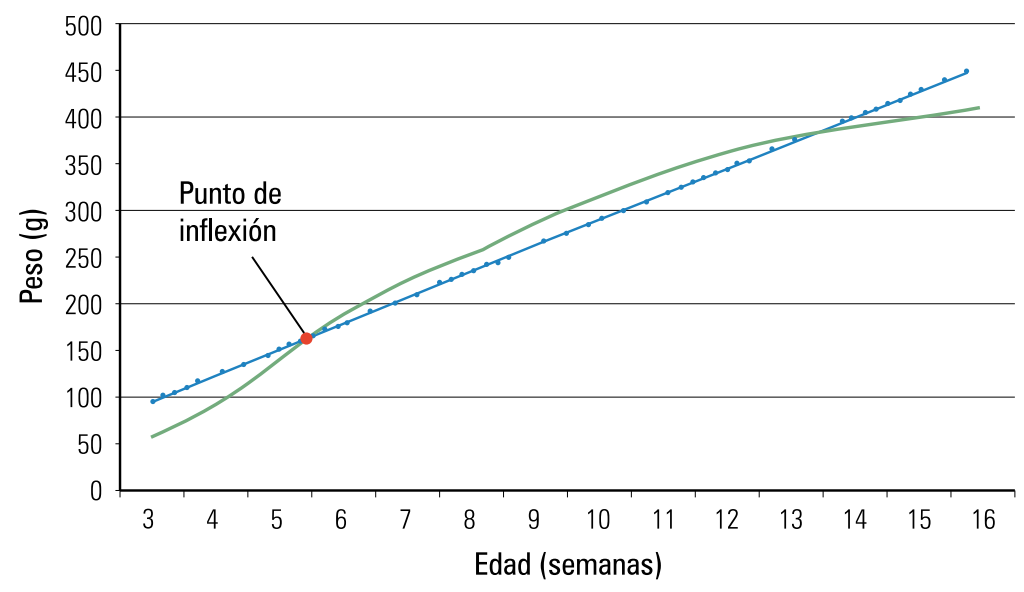

Figura 1. Curva de crecimiento longitudinal de la masa corporal (g) de ratas machos Wistar desde la tercera hasta la decimosexta semana de vida. 


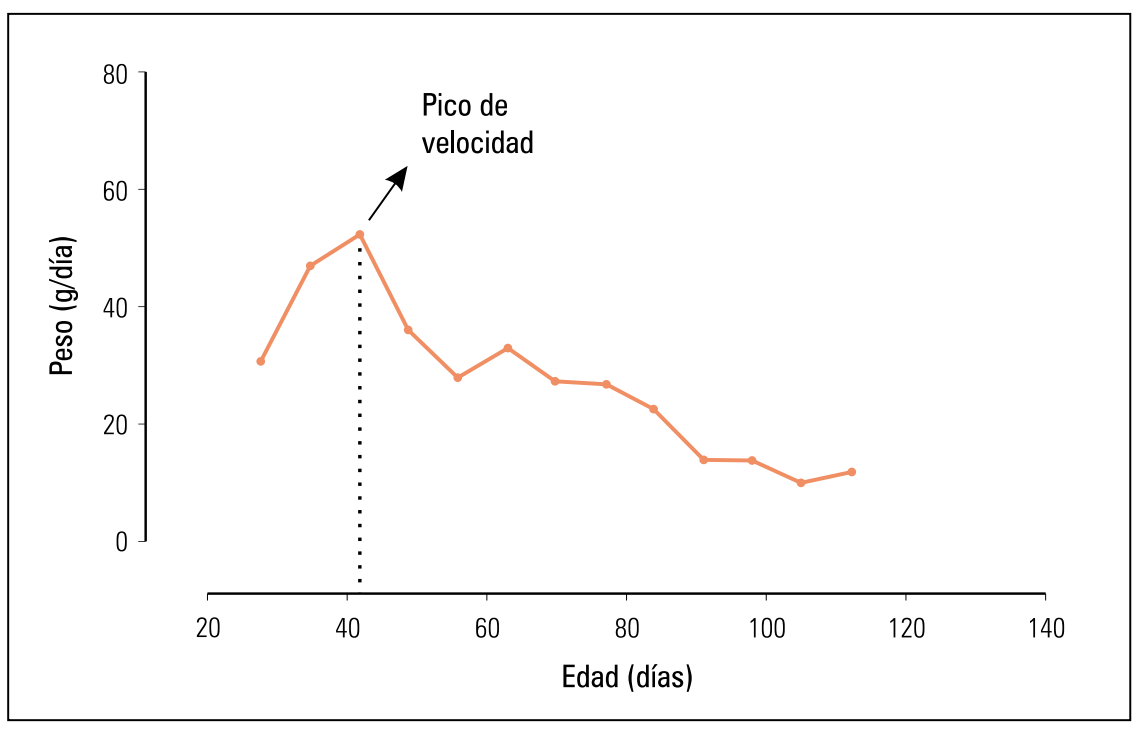

Figura 2. Pico de velocidad de crecimiento (PHV) de ratas machos Wistar, en función de la edad.

21 días (14,6\%) hasta los 112 días de vida $(100 \%)$. Estos valores permiten distinguir tres fases durante el proceso de crecimiento: una fase de crecimiento lento al inicio (pre-PVC), seguido de una fase de crecimiento acelerado (PVC) y una última fase de desaceleración del crecimiento (post-PVC).
Con el propósito de analizar la correlación entre las variables de edad, masa corporal y pico de velocidad de crecimiento, se verificó que los coeficientes " $r$ " de Pearson fueran significativos en todos los casos ( $p<0,001)$; estos valores pueden ser observados en la figura 3.

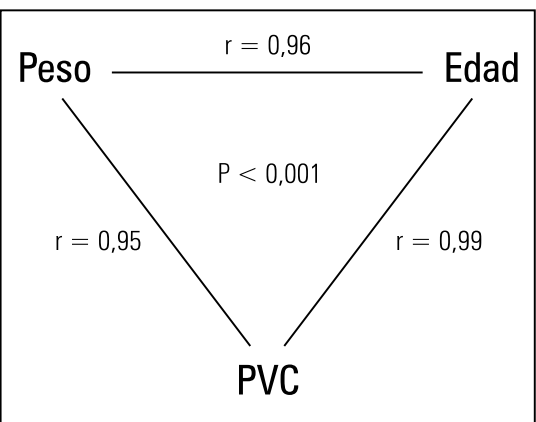

Figura 3. Correlación entre variables murinométricas.

Por otro lado, para establecer una relación matemática entre la variable dependiente de pico de velocidad de crecimiento (PVC) y las variables independientes de masa corporal (g) y edad (días), se realizó el análisis de regresión lineal simple y múltiple. En este sentido, la masa corporal de forma individual explica un $89 \%$, la edad un $99 \%$, y cuando se analizó con ambas variables mediante el análisis de regresión múltiple los resultados evidencian $99 \%$, por lo que ambas variables independientes son determinantes para la predicción

Tabla 2. Valores medios $(\mathrm{X})$, desviación estándar $(\mathrm{DE})$, delta $(\Delta)$ y velocidad de crecimiento $(\mathrm{VC})$ de la masa corporal de ratas machos durante 14 semanas de monitorización.

\begin{tabular}{|c|c|c|c|c|c|c|c|}
\hline \multirow{2}{*}{$\begin{array}{l}\text { Número de } \\
\text { semanas } \\
\text { evaluadas }\end{array}$} & \multirow{2}{*}{$\begin{array}{c}\text { Edad } \\
\text { semanas }\end{array}$} & \multirow{2}{*}{ Edad días } & \multirow{2}{*}{$(\Delta)$} & \multicolumn{2}{|c|}{ Masa corporal $(\mathrm{g})$} & \multirow{2}{*}{$\begin{array}{c}\text { Porcentaje de } \\
\text { crecimiento } \\
\%\end{array}$} & \multirow{2}{*}{$V C(g / d)$} \\
\hline & & & & $\mathrm{x}$ & $\mathrm{DE}$ & & \\
\hline $1^{\mathrm{a}}$ & 3 & 21 & -- & 60,16 & 7,46 & 14,60 & -- \\
\hline $2^{\mathrm{a}}$ & 4 & 28 & 30,63 & 90,80 & 9,74 & 22,00 & 4,20 \\
\hline $3^{\mathrm{a}}$ & 5 & 35 & 47,00 & 137,76 & 15,58 & 33,40 & 6,90 \\
\hline $4^{a}$ & 6 & 42 & 52,50 & 190,24 & 15,61 & 46,00 & 7,50 \\
\hline $5^{a}$ & 7 & 49 & 35,70 & 225,96 & 23,42 & 54,70 & 5,30 \\
\hline $6^{\mathrm{a}}$ & 8 & 56 & 27,80 & 253,72 & 21,24 & 61,50 & 3,90 \\
\hline $7^{\mathrm{a}}$ & 9 & 63 & 32,90 & 286,60 & 18,00 & 69,30 & 4,60 \\
\hline $8^{\mathrm{a}}$ & 10 & 70 & 27,30 & 313,92 & 18,49 & 76,10 & 3,90 \\
\hline$g^{a}$ & 11 & 77 & 27,10 & 341,00 & 22,30 & 82,60 & 3,80 \\
\hline $10^{\mathrm{a}}$ & 12 & 84 & 22,60 & 363,64 & 22,41 & 88,10 & 3,30 \\
\hline $11^{\mathrm{a}}$ & 13 & 91 & 13,60 & 377,28 & 24,41 & 91,40 & 2,00 \\
\hline $12^{\mathrm{a}}$ & 14 & 98 & 13,70 & 390,96 & 22,29 & 94,50 & 1,90 \\
\hline $13^{\mathrm{a}}$ & 15 & 105 & 10,10 & 401,04 & 21,53 & 97,20 & 1,40 \\
\hline $14^{\mathrm{a}}$ & 16 & 112 & 11,70 & 412,77 & 22,22 & 100,00 & 1,70 \\
\hline
\end{tabular}

$(p<0,001)$ Diferencias significativas de masa corporal entre todas las edades (ANOVA medidas repetidas). $\mathrm{VC}=$ velocidad de crecimiento, $\mathrm{DE}=$ desviación estándar 
Tabla 3. Valores de regresión lineal para la predicción del pico de velocidad de crecimiento (PVC) a partir de la edad y la masa corporal.

\begin{tabular}{|c|c|c|c|c|c|c|}
\hline \multirow{2}{*}{ Modelo } & \multicolumn{2}{|c|}{ B } & \multirow{2}{*}{ C } & \multirow{2}{*}{$\mathrm{R}$} & \multirow{2}{*}{$\mathrm{R}^{2}$} & \multirow{2}{*}{$\mathrm{p}$} \\
\hline & Edad & Masa corporal & & & & \\
\hline 1 & 0,133 & -- & $-5,05$ & 0,99 & 0,99 & $p<0,0001$ \\
\hline 2 & -- & 0,0306 & $-4,70$ & 0,95 & 0,89 & $p<0,0001$ \\
\hline 3 & 0,143 & 0,00259 & $-5,02$ & 0,99 & 0,99 & $p<0,0001$ \\
\hline
\end{tabular}

Tabla 4. Ecuaciones de regresión para estimar el pico de velocidad de crecimiento (PVC) a partir de la edad y la masa corporal.

\begin{tabular}{cccc} 
Modelo & Ecuación & $\mathrm{R}^{2}$ & EEE \\
1 & $\mathrm{PVC}=-5,05+\left(0,133^{*}\right.$ Edad $)$ & 0,99 & 0,35 \\
2 & $\mathrm{PVC}=-4,70+\left(0,030{ }^{*}\right.$ Peso $)$ & 0,89 & 1,18 \\
3 & $\mathrm{PVC}=-5,02+\left(0,143{ }^{*}\right.$ Edad $)-(0,00259 *$ Peso $)$ & 0,99 & 0,34 \\
\hline
\end{tabular}

Edad (días); Peso (g); EEE = error estándar de estimación. del pico de velocidad de crecimiento en ratas machos Wistar (ver tabla 3 ).

Las ecuaciones de regresión lineal pueden ser observadas en la tabla 4 , donde el modelo 1 y 3 son los que muestran mayor grado de homogeneidad (EEE $=0,34-0,35)$ en relación al modelo $2(\mathrm{EEE}=1,18)$, puesto que presentan menor error estándar. A fin de comprobar la validez de las mismas, se comparó los datos reales (originales) del estudio con los valores predichos, donde no se observó diferencias significativas ( $p>0,001$ ); inclusive, el plotaje de Bland y Altman mostró alta concordancia entre los valores reales y predichos, donde la diferencia de las medias entre las dos mediciones resultó de la siguiente forma: media del PVC real con media del PVC predicho a través del peso $(-0,01)$, media del PVC real con media del PVC predicho a través de la edad $(-0,02)$ y media del PVC real con media del PVC predicho a través del peso y edad $(0,004)$ (ver figura 4$)$.

Los valores obtenidos por medio de las ecuaciones de regresión fueron distribuidos en intervalos, a través del cual se puede clasificar el PVC en 14 niveles, los cuales indican la proximidad y el alejamiento del PVC. Estos valores son detallados en la tabla 5 , donde $-0,5 \leq$ $\mathrm{x}<0,5$ es el intervalo que indica el mo- mento exacto del PVC; cuando el valor es $\geq 9,5$ indica que el proceso de crecimiento somático llegó a su fase final, por lo que las ratas serían consideradas como adultos jóvenes.

\section{DISCUSIÓN}

En estudios de crecimiento y desarrollo es importante distinguir entre el tamaño absoluto que un animal alcanzó en una edad y en una determinada fase de maduración ${ }^{(21)}$, puesto que los cambios biológicos ocurren de forma secuencial y ordenada durante la vida ${ }^{(22)}$ y llevan al ser viviente a alcanzar el estado adulto ${ }^{(1,23,24)}$ siempre que las condiciones nutricionales ${ }^{(25)}$ y medio ambientales sean normales y lo permitan. De hecho, los factores intrínsecos y extrínsecos tienen un importante papel y condicionan el crecimiento somático de los animales y su desarrollo dependerá del potencial genético y su capacidad de adaptación al medio ambiente. Desde esa perspectiva, podemos señalar que existen varios tipos de maduración biológica, como somática, esquelética, dental y sexual ${ }^{(22)}$ y cada uno de estos tipos permiten distinguir una subclasificación, donde se puede identificar el grado de maduración biológica. Para tal efecto, el presente estudio utilizó la evaluación somática de la masa corporal con el objetivo de determinar la velocidad de crecimiento (VC) y consecuentemente identificar el pico de velocidad de crecimiento (PVC) y proponer ecuaciones de regresión para predecir el PVC, respectivamente. Los resultados del presente estudio pusieron en evidencia que el PVC fue alcanzado aproximadamente a los 42 días de vida, lo que representó el $46 \%$ del crecimiento somático respecto
Tabla 5. Clasificación del Pico de Velocidad del Crecimiento (PVC).

\begin{tabular}{ccc} 
Nivel & Intervalos considerados & Indicador \\
-3 & $\mathrm{X} \leq-2,5$ & Por debajo del PVC en tres semanas \\
-2 & $-2,5 \leq \mathrm{x}<-1,5$ & Por debajo del PVC en dos semanas \\
-1 & $-1,5 \leq \mathrm{x}<-0,5$ & Por debajo del PVC en una semana \\
0 & $-0,5 \leq \mathrm{x}<0,5$ & Momento exacto del PVC \\
1 & $0,5 \leq \mathrm{x}<1,5$ & Por encima del PVC en una semana \\
2 & $1,5 \leq \mathrm{x}<2,5$ & Por encima del PVC en dos semanas \\
3 & $2,5 \leq \mathrm{x}<3,5$ & Por encima del PVC en tres semanas \\
4 & $3,5 \leq \mathrm{x}<4,5$ & Por encima del PVC en cuatro semanas \\
5 & $4,5 \leq \mathrm{x}<5,5$ & Por encima del PVC en cinco semanas \\
6 & $5,5 \leq \mathrm{x}<6,5$ & Por encima del PVC en seis semanas \\
7 & $6,5 \leq \mathrm{x}<7,5$ & Por encima del PVC en siete semanas \\
8 & $7,5 \leq \mathrm{x}<8,5$ & Por encima del PVC en ocho semanas \\
9 & $8,5 \leq \mathrm{x}<9,5$ & Por encima del PVC en nueve semanas \\
10 & $\mathrm{X} \geq 9,5$ & Fase final del crecimiento somático \\
\hline
\end{tabular}




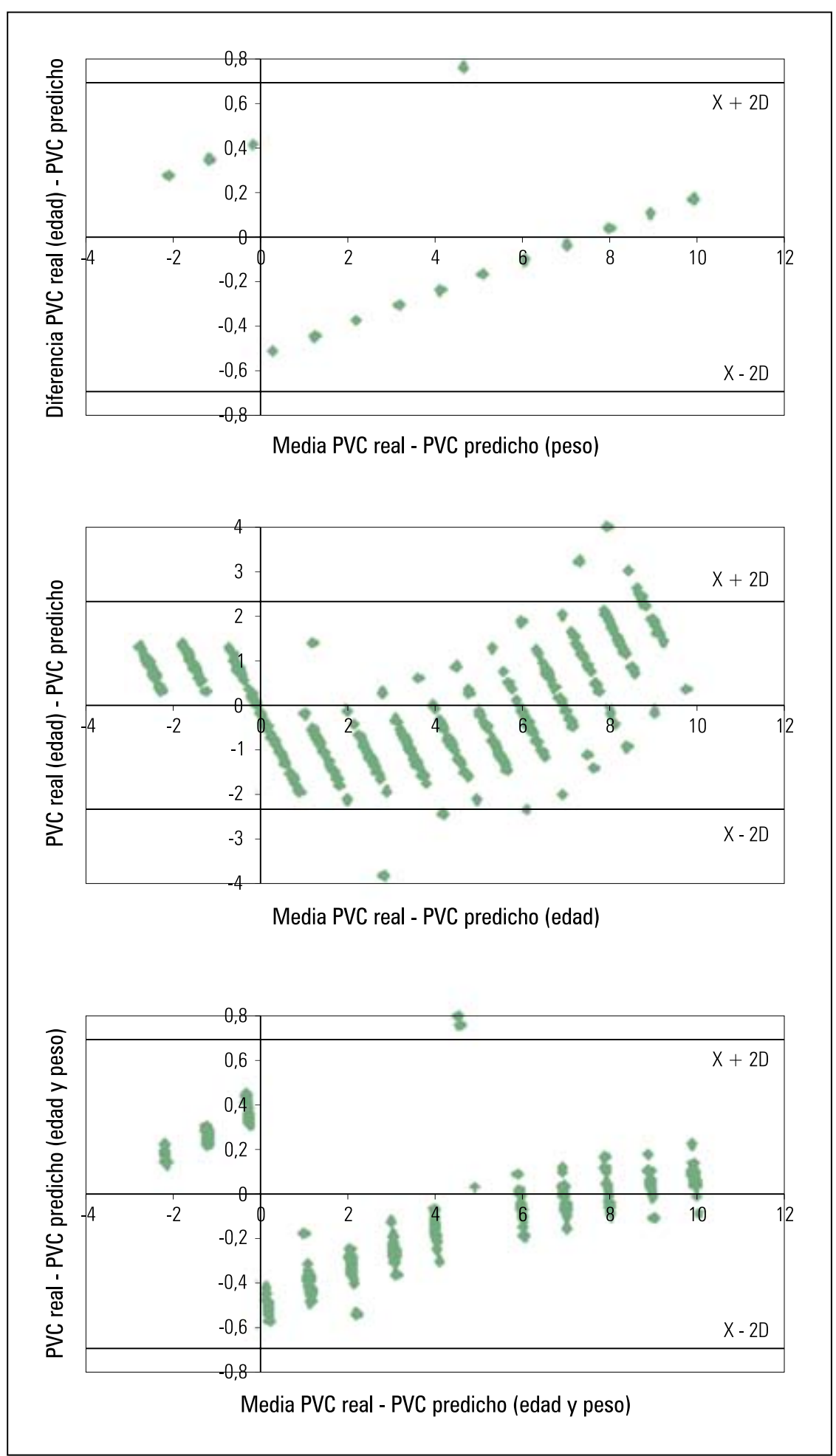

Figura 4. Concordancia entre los valores reales del PVC y los valores predichos, por medio del plotaje de Bland y Altman. a la masa corporal de la etapa adulta. Evidentemente, estos hallazgos se encuentran en el rango del rápido proceso de crecimiento que las ratas Wistar muestran y que se suele observar según la literatura desde los 25 hasta los 70 días de edad ${ }^{(26)}$. Este hecho puede ser observado en la figura 2, donde el primer pico muestra un mayor aumento de porcentaje del crecimiento somático ( $\mathrm{g} /$ semana) en relación al segundo, lo que demuestra que en ese transcurso los animales experimentaron un rápido proceso de crecimiento somático, seguido de una fase de desaceleración y estabilización, respectivamente. Respecto a las investigaciones que podrían justificar de mejor forma los resultados del presente estudio, destacamos que no se encontró evidencias que permitieran contrastar con nuestros resultados, salvo el estudio de Willians y col ${ }^{(27)}$, que describen el PVC en ratas Wistar a los 55 días, tanto en ratas eutróficas, como con retraso. Esta diferencia de aproximadamente de dos semanas, probablemente se deba al fenómeno de la tendencia secular ocurrida a lo largo de los años, lo que podría explicar en parte la maduración precoz de las ratas del presente estudio. Sin embargo, en relación a la maduración sexual varios estudios muestran que las ratas Wistar alcanzan la maduración aproximadamente a los 50 días de vida ${ }^{(8,28)}$ y 48 días ${ }^{(29)}$, siendo estos valores relativamente mayores en una semana en comparación con los del presente estudio. A su vez, Hughes y Tanner ${ }^{(21)}$, al investigar la maduración esquelética de ratas de la misma cepa, verificaron el PVC a los 33 días de vida. Por lo tanto, estos valores evidenciados de forma general varían aproximadamente de una a dos semanas entre los estudios, probablemente debido a la técnica utilizada y/o a factores intrínsecos y extrínsecos que podrían afectar el proceso normal de crecimiento y desarrollo somático. Según Dixon y Styrt ${ }^{(26)}$, las ratas machos parecieran crecer a una velocidad máxima durante al menos los primeros 60 días de vida y, según Meisami ${ }^{(28)}$, hasta los 70 días de vida, respectivamente. Cabe resaltar que el crecimiento esquelético termina 
alredor de los 120 días en ratas machos y hembras ${ }^{(21)}$, llegando de esta forma a la adultez, y consecuentemente a la estabilización del crecimiento somático.

Por otro lado, durante el proceso de monitorización del peso corporal, la curva de crecimiento somático sugiere cambios morfológicos desde el periodo del destete de 21 días hasta los 112 días de vida, aproximadamente, por lo que se puede distinguir tres fases de crecimiento: inicial, intermedia y final (2). Estas fases deben ser ampliamente conocidas y consideradas por los investigadores que trabajan con modelos animales, puesto que muchos estudios evalúan un sinnúmero de variables intrínsecas y extrínsecas en ratas de laboratorio en edades inferiores a los 100 días. De hecho, tales animales no podrían ser considerados como adultos según algunas investigaciones ${ }^{(21,27)}$ y los resultados obtenidos en el presente estudio.

En consecuencia, los estudios experimentales que engloban tratamientos y/o intervenciones de una semana y/o más tiempo, y seleccionan ratas con edad inferior a los 100 días deben tomar en cuenta el pico de velocidad de crecimiento (PVC). Para tal efecto, en nuestro estudio proponemos tres ecuaciones de regresión lineal, los cuales muestran altos valores predictivos $\left(\mathrm{R}^{2}=0,89-0,99\right)$ y concordancia entre los valores reales y estimados. Estas ecuaciones permiten predecir la fase sensible y/o crítica de los roedores a través del uso de variables como la edad y el peso corporal.

Por medio de las regresiones lineales es posible predecir tres fases del crecimiento somático que fueron descritas anteriormente por Prieto y Álvarez ${ }^{(5)}$, donde la primera fase es caracterizada por la lentitud del crecimiento, correspondiendo en el presente estudio al intervalo $\mathrm{X}<-0,5$ (nivel -3 a -1 ); la segunda fase de crecimiento es considerada como aceleración máxima, que corresponde al intervalo $-0,5 \leq$ $\mathrm{x}<0,5$ (nivel 0 ); y la tercera y última fase, denominada de desaceleración y estabilización (fase final), es equivalente al intervalo $x \geq 0,5$ (nivel 1-10) del presente estudio. Esta distinción de niveles permite identificar el grado de maduración somática de las ratas Wistar, puesto que la edad es una variable importante en los diferentes mecanismos de regulación y en el control de las distintas funciones biológicas, así como la masa corporal es decurrente de la actividad metabólica ${ }^{(13)}$. Por lo tanto, estas evidencias hacen suponer que es necesario de herramientas no-invasivas que permitan determinar la fase sensible y crítica de las ratas Wistar. De esta forma, identificar a los animales que se encuentren más próximos y distantes al pico de velocidad de crecimiento es de interés para quienes trabajan con modelos animales inferiores a los 112 días de vida, ya que, en la fase sensible, los roedores experimentan cambios biológicos significativos que los investigadores deberían tomar en consideración. En este sentido, a la hora de analizar los resultados, las variables evaluadas podrían ser influidas y contaminadas por efectos del crecimiento somático y la maduración biológica, y no por efectos de las intervenciones y/o tratamientos experimentales. Por ello, consideramos necesario el uso de criterios no-invasivos para identificar la fase sensible y crítica de los animales y controlar minuciosamente las variables extrañas durante los experimentos, los cuales son considerados como fuentes de invalidación interna.

Con relación a las limitaciones del estudio, no se pudo controlar algunas variables como la medición de la longitud total de las ratas, evaluación del cartílago de crecimiento mediante rayos X y el análisis de parámetros bioquímicos que hubieran permitido justificar de mejor forma los resultados del presente estudio. Tales limitaciones deben ser tomadas en consideración a la hora de analizar los resultados, por lo que sugerimos evaluar las variables antes mencionadas en futuras investigaciones y contrastar con nuestros resultados.
En relación a las ecuaciones propuestas, a pesar de cumplir con los estándares mínimos de aceptación, su posible aplicación a otras cepas podría generar imprecisión en los resultados, por lo que su uso se limita a ratas machos Wistar.

En resumen, la gran similitud entre los genomas de los roedores con los humanos ${ }^{(6)}$ es aprovechada por los investigadores que trabajan con modelos humanos ${ }^{(7)}$ en las áreas de nutrición ${ }^{(11)}$, endocrinología, farmacología, bioquímica, entre otras. Por lo que es necesario considerar las diferencias en cuanto a su anatomía, fisiología y desarrollo biológico de los roedores ${ }^{(30)}$, esto con la intención de buscar la especificidad en el momento de extrapolar los resultados del modelo animal al modelo humano.

A través de los resultados encontrados en el presente estudio, se concluye que el PVC de ratas machos Wistar analizado a partir de la masa corporal se presentó a los 42 días de vida. A su vez, para determinar el grado de maduración somática se generó tres ecuaciones de regresión lineal que permitieron predecir el PVC a partir de la edad y la masa corporal. Los resultados de dichas ecuaciones pueden ser interpretadas a través de 14 niveles que especifican la proximidad y el alejamiento del PVC, por lo que su uso en este tipo de cepa podría ayudar a seleccionar grupos específicos de investigación, puesto que implica simplicidad, costo bajo y es considerado como un método no-invasivo.

\section{REFERENCIAS BIBLIOGRÁFICAS}

1. Malina RM, Bouchard C, Bar-Or O. Growth maturation and physical activity. 2nd ed, Champaign: Human Kinetics Books; 2004.

2. Enesco M, Leblond $P$. Increase in cell number as factor in the growth of the organs and tissues of the young male rat. J Embryol Exp Morph. 1962;10(4):530-62.

3. Layman DK, Hegarty PVJ, Swan PB. Comparison of morphological and biochemical parameters of growth in rat skeletal muscles. J Anat. 1980;130(1):159-71

4. Roche A. Bone Growth and maturation. En: FaIkner F, Tanner JM, Editors. Human Growth. A comprehensive treatise. New York: Plenum Press; 1982:25-59. 
5. Prieto Fernández JG, Álvarez de Felipe AL. Fisiología comparada de los animales de experimentación. En: Pérez Garcia CC, Diez Prieto MI, Garcia Partida P. (editores). Introducción a la experimentación y protección animal. León: Universidad de León; 1999:91-102.

6. Diemen D, Trindade E, Trinidade M. Experimenta model to induce obesity in rats. Acta Cir Bras 2006;21(6):425-9.

7. Harari D, Hermolin G, Harari O. The effect of age on morphology and eruption of the lower incisors in mature rats. Arch Oral Biol. 2005;50(11):953-8.

8. Lee, VWK, De Kretser DM, Hudson B, Wang C Variations in serum FSH, LH and testosterone levels in male rats from birth to sexual maturity. J Reprod Fert. 1975;42(1):121-6.

9. Popp, MB, Seoras MD, Morrison D, Brennan F Growth and body composition during long-term total parenteral nutrition in the rat. Am J Clin Nutr. 1982;36:1119-28.

10. Goodgame JT, Lowry SF, Brennan MF. Body weigh change and nutritional adequacy in the parenterally alimented rat. J Surg Res. 1978;24(6):520-6.

11. Mazeti CM, Monserrat, Furlán MMDP. Crescimento e parâmetros reprodutivos de ratas wistar, em restrição alimentar desde o nascimento. Acta Sci Biol Sci Maringá. 2008;30(2):197-204.

12. Canteras-Jordana M. Diseño de experimentos con animales de laboratorio. En: Rodríguez Martínez J, Hernández Lorente MD, Costa Ruiz J. (Editores). Introducción a la experimentación con animales. Murcia: Universidad de Murcia; 2001:21-8.

13. Llinás MT, González JD, Moreno C, Salazar FJ. Modelos animales experimentales. En: Rodríguez Martínez J, Hernández Lorente MD, Costa Ruiz J. (Editores). Introducción a la experimentación con animales. $1^{a}$ edición. Universidad de Murcia, 2001:85-94.

14. Thomas J, Nelson J. Research Methods in Physi- cal Activity. Champaign: Human Kinetics Books. 1996.

15. Glenn ND. Cohort analysis. Beverly Hills: Calif: Sage Publications Series. Quantitative applications in the Social Sciences. 1977.

16. Cossio-Bolanos MA, Gomez Campos RA, Arruda M, Fogaça RT. Valores de confiabilidad de indicadores somáticos en ratas machos wistar. Actualización en Nutrición. 2011:11(4):296-302.

17. Cossio-Bolanos MA, Gomez Campos RA, Fogaça RT, Arruda M, Efectos de la suplementación de vitamina $\mathrm{E}$ sobre la fatiga muscular en ratas entrenadas y sedentárias. Nutr clín diet hosp. 2011;31(2):34-40.

18. Gomez Campos RA, Arruda M, Borges F, CossioBolanos MA. Effects of supplementation of Lcarnitine on the body composition of trained and sedentary rats. Biomecânica. 2010;18(2):7-13.

19. Bland JM, Altman DG. Statistical methods for assessing agreement between two methods of clinical measurement. Lancet. 1986;1:307-10.

20. Fox E, Shotton K, Ulrich C. Sigma-Stat User's Manual (cd), San Rafael, CA., (USA): Jandel Scientific Co, 1995.

21. Hughes PCR, Tanner JM. The assessment of skeletal maturity in the growing rat. J Anat. 1970;106(2):371402.

22. Martin RH, Uezu R, Parra S, Arena S, Bojikian L, Böhme M. Auto-avaliação da maturação sexual masculina por meio da utilização de desenhos e fotos. Rev Paul Educ Fis São Paulo. 2001;15(2):212-22.

23. Duarte MFS. Maturação Fisica: Uma Revisão da Literatura, com Especial Atenção à Criança Brasileira. Caderno de Saúde Pública (Rio J). 1993;9(1):71-84.

24. Chipkovitch E. Avaliação clínica da maturação sexual na adolescência Clinical assessment of sexual maturation in adolescents .J Pediatr (Rio J). 2001;77(2):135-42
25. Alves A, Damaso A, Damaso $V$. The effects of prenatal and postnatal malnutrition on the morphology, differentiation, and metabolism of skeletal striated muscle tissue in rats. Jornal de Pediatria. 2008:84(3):264-71.

26. Dixon AD, Styrt PJ. Growth changes in the craniofacial complex of the rat after prolonged papa in administration. Am J Orthod Dentofac Orthop. 1994;105:270-8.

27. Willians JPG, Tanner JM, Hughes PCR. Catch-up growth in male rats after growth retardation during the suckling period. Pediat Res. 1974;8(3):149-56.

28. Meisami E. Complete recovery of growth deficits after reversal of PTU-induced postnatal hypothyroidism in the female rat: a model for catch-up growth Life Sci. 1984;34:1487-96.

29. Clermont $Y$. Kinetics of spermatogenesis in mammals: somniferous epithelium cycle and spermatogonial renewal. Physiol Rev. 1972;52(1):198-236

30. Quinn DVM. Comparing rats to human's age: How old is my rat in people years. Nutrition. 2005;21(6):775-7.

Artículo recibido el 23 de setiembre de 2011 y aceptado para publicación el 10 de enero de 2012.

\section{Correspondencia:}

Prof. Dr. Marco Cossio Bolaños

Universidade Estadual de Campinas

Faculdade de Educação Física

Av. Erico veríssimo 701, Cidade Universitária 13083-851

Caixa Postal 6134. Campinas, São Paulo, Brasil.

Correo electrónico: mcossio1972@hotmail.com 